\title{
Myocardial stress perfusion scintigraphy for outcome prediction in patients with severe left ventricular systolic dysfunction
}

\author{
Alessia Gimelli ${ }^{1}$ (D) Alberto Aimo ${ }^{1,2} \cdot$ Emilio Maria Pasanisi ${ }^{1} \cdot$ Michele Alessandro Coceani $^{1} \cdot$ Alberto Clemente $^{1}$. \\ Michele Emdin ${ }^{1,2} \cdot$ Leslee J. Shaw ${ }^{3}$
}

Received: 15 January 2021 / Accepted: 9 March 2021

(C) Springer-Verlag GmbH Germany, part of Springer Nature 2021

\begin{abstract}
Coronary angiography has been recommended in all patients with suspected chronic coronary syndrome and left ventricular ejection fraction $(\mathrm{LVEF}) \leq 35 \%$. The role of ischemia testing, for example, through stress-rest myocardial perfusion scintigraphy (MPS), for risk prediction is not well established.
\end{abstract}

\section{Methods}

We evaluated 1576 consecutive patients referred to MPS and stratified into 3 LV ejection fraction (LVEF) categories: $\leq 35 \%, 36-$ $49 \%$, and $\geq 50 \%$.

\section{Results}

Patients with LVEF $\leq 35 \%$ were oldest, most often men, and with the highest likelihood of prior early (elective or urgent) coronary revascularization. They had also the highest values or summed stress score (SSS), summed rest score (SRS), and summed difference score (SDS), as well as the highest frequency of significant coronary artery disease, and a greater number of diseased vessels. Follow-up: In this subgroup, 32 cardiovascular death or non-fatal myocardial infarction (MI) (21\%), 35 allcause deaths (22\%), and 37 cardiovascular deaths, non-fatal MI, or late revascularizations (27\%) were recorded with the shortest survival among all LVEF classes. SRS, SSS, and SDS had very low area under the curve values for the prediction of the 3 endpoints, with very high cut-offs, respectively. SRS and SSS cut-offs predicted a worse outcome in Cox regression models including the number of diseased vessels and early revascularization.

\section{Conclusions}

In patients with LVEF $\leq 35 \%$, SRS and SSS are less predictive of outcome than in patients with better preserved systolic dysfunction, but their cut-offs retain independent prognostic significance from the number of vessels with significant stenoses and from early revascularization.

Keywords Left ventricular ejection fraction · LV systolic dysfunction · Coronary artery disease

This article is part of the Topical Collection on Cardiology

Alessia Gimelli and Alberto Aimo equally contributed.

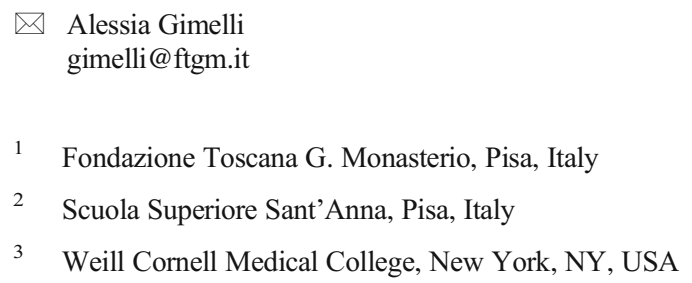

\begin{tabular}{ll}
\hline & $\begin{array}{l}\text { Alessia Gimelli } \\
\text { gimelli@ftgm.it }\end{array}$ \\
1 & Fondazione Toscana G. Monasterio, Pisa, Italy \\
2 & Scuola Superiore Sant'Anna, Pisa, Italy \\
3 & Weill Cornell Medical College, New York, NY, USA
\end{tabular}

\section{Introduction}

Individuals with left ventricular (LV) systolic dysfunction may have signs and symptoms of heart failure (HF), or be currently asymptomatic but with a higher risk of developing $\mathrm{HF}$ [1]. Extensive coronary artery disease (CAD) and previous myocardial infarction (MI) are leading causes of LV systolic dysfunction. In this setting, the role of inducible ischemia as a risk predictor and as a guide to treatment is uncertain [2]. In the Surgical Treatment for Ischemic Heart Failure (STICH) trial, patients with left ventricular ejection fraction (LVEF) 
$\leq 35 \%$ and inducible ischemia did not have a shorter survival nor a greater benefit from coronary artery bypass grafting (CABG) than the other patients [3]. Accordingly, the latest European Society of Cardiology guidelines recommended to consider coronary revascularization in all patients with chronic coronary syndrome (CCS) and LVEF $\leq 35 \%$ regardless of the presence and extent of myocardial ischemia [4]. This recommendation might further accentuate a tendency to skip the search for ischemia in patients with HF for reasons including the frequency of submaximal tests because of beta-blocking therapy or premature exercise discontinuation, a higher risk of ventricular arrhythmias, and longer examination times than a simple assessment of myocardial viability.

In this study, we reappraised the role of ischemia testing in patients with LV systolic dysfunction by examining the prognostic value of perfusion deficits at rest and at peak stress on myocardial perfusion scintigraphy (MPS), in a large cohort of patients spanning across the spectrum of LVEF and with longterm follow-up data.

\section{Methods}

\section{Patient population}

The Analysis of Myocardial Ischemia by Cadmium-zinc-telluride: accuracy and Outcome (AMICO) study was a prospective, non-randomized study conducted between January 2010 and June 2019. We prospectively enrolled consecutive patients referred to stress-rest MPS with a cadmium zinc telluride (CZT) camera at the nuclear cardiology laboratory of the Fondazione Toscana Gabriele Monasterio (FTGM) in Pisa, Italy $(n=1576)$. All patients underwent a thorough clinical characterization within 1 month from MPS and an assessment of coronary anatomy by CCTA and/or invasive coronary angiography (ICA) [5-8]. Patients with abnormal MPS underwent ICA, while those with a minimally abnormal or uncertain MPS (123 patients, 8\%) result underwent CCTA and were referred to ICA whether CCTA had shown or could not exclude obstructive CAD (8/123 patients). Patients underwent coronary revascularization in the following cases: (1) coronary artery diameter stenosis $\geq 70 \%$ and evidence of inducible ischemia in the same territories from MPS or invasive measures (fractional flow reserve $\leq 0.80$ ), (2) stenoses $>90 \%$, (3) $\geq 50 \%$ left main coronary (LMC) stenosis, (4) LVEF $\leq 35 \%$ attributed to CAD. Patients with CCS also received OMT [4]. We excluded patients with acute or recent ( $<3$ months) MI, unstable angina, non-ischemic cardiomyopathy, moderate-to-severe heart valve disease, end-stage renal disease, or active malignancy. According to the aim of this study, patients were stratified in the following LVEF categories: $\geq 50 \%$ (i.e., the cut-off for systolic dysfunction), 36-49\%, and $\leq 35 \%$ (the threshold for device implantation) $[9,10]$. In agreement with the entry criteria of the STICH trial, HF signs and symptoms were not considered for the present analysis [11].

All participants gave written informed consent. The study conformed to the declaration of Helsinki and was approved by the Institution's human research committee.

\section{Myocardial perfusion scintigraphy}

The MPS protocol was published previously $[5,6,8]$. In all patients with previous MI, nitrates were administered before rest injection, to optimize the viability evaluation $[12,13]$. Stress and rest perfusion images from the CZT camera were semi-quantitatively scored according to the 17 -segment LV model [14] and a 5-point scale ( 0 normal, 1 equivocal, 2 moderate, 3 severe reduction of tracer uptake, 4 absence of tracer uptake). The summed stress score (SSS), summed rest score (SRS), and summed difference score (SDS) were calculated. The percent of LV myocardium with stress, fixed, or ischemic defects can be calculated as SSS, SRS, and SDS/68 (maximum potential score $=4 * 17) \%$, respectively. LV volumes, $\mathrm{EF}$, and mass were measured after stress and at rest using the Corridor 4DM-SPECT (4DM) software [15]. The same software automatically fits the LV volume curve with a fourth-order harmonic function to derive the peak filling rate (end-diastolic volume/s) as an indicator of LV diastolic function [16]. The readers were blinded to clinical data and coronary anatomy.

As per protocol, myocardial perfusion during stress was defined normal or minimally abnormal by SSS $<4$ and moderately/severely abnormal by SSS $>7$ (involving $>10 \%$ of LV myocardium) [17]. Reversible ischemia was defined as $\operatorname{SDS} \geq 4$.

\section{Coronary computed tomography angiography}

CCTA was performed on a 64-slice scanner (GE Discovery VCT; GE Healthcare) (up to January 2013) or a 320-slice scanner (Aquilon one, Toshiba). Stenosis severity was measured on multiplanar reformatted images using an automatic interactive program to quantify coronary luminal narrowing [18]. Obstructive CAD was defined by the presence of $\geq 70 \%$ luminal diameter reduction in the epicardial coronary arteries or $\geq 50 \%$ in the LMC.

\section{Invasive coronary angiography and revascularization}

ICA was performed using standard techniques (Innova 2000 GE; General Electric). Coronary angiograms were quantitatively analyzed using an off-line computer-based software program (MEDIS CMS version 6.0; MEDIS Imaging Systems) with an automatic edge-contour detection algorithm. Significant stenoses were defined as above. 
Table 1 Clinical variables and risk factors

\begin{tabular}{|c|c|c|c|c|}
\hline & $\begin{array}{l}\mathrm{LVEF} \leq 35 \% \\
n=156(10 \%)\end{array}$ & $\begin{array}{l}\text { LVEF } 36-49 \% \\
n=207(13 \%)\end{array}$ & $\begin{array}{l}\mathrm{LVEF} \geq 50 \% \\
n=1213(77 \%)\end{array}$ & $p$ \\
\hline Age (years) & $72(65-77)$ & $71(65-77)$ & $70(62-76)$ & 0.045 \\
\hline Men, $n(\%)$ & $136(87)$ & $180(87)$ & $856(71)$ & $<0.001$ \\
\hline NYHA class I, II, III, $n(\%)$ & $1003,209,1(83,17,0)$ & $136,70,1(66,34,0)$ & $42,96,18(2762,12)$ & $<0.001$ \\
\hline Typical angina, $n(\%)$ & $45(29)$ & $85(41)$ & $600(49)$ & $<0.001$ \\
\hline Pre-test probability $<5 \%, 5-15 \%,>15 \%, n(\%)$ & $0,2,104(0,1,67)$ & $0,8,188(0,4,91)$ & $0,73,1077(0,6,89)$ & 0.095 \\
\hline Family history of CAD, $n(\%)$ & $68(44)$ & $82(40)$ & $574(47)$ & 0.092 \\
\hline Previous PCI, $n(\%)$ & $48(31)$ & $49(24)$ & $148(12)$ & $<0.001$ \\
\hline Previous MI, $n(\%)$ & $81(52)$ & $63(30)$ & $129(11)$ & $<0.001$ \\
\hline Current or former smoker, $n(\%)$ & $34(22)$ & $61(29)$ & $329(27)$ & 0.246 \\
\hline Diabetes, $n(\%)$ & $66(42)$ & $81(39)$ & $446(37)$ & 0.371 \\
\hline Hypertension, $n(\%)$ & $97(62)$ & $121(58)$ & $713(59)$ & 0.719 \\
\hline Hypercholesterolemia, $n(\%)$ & $72(46)$ & $93(45)$ & $595(49)$ & 0.457 \\
\hline CKD stage $4-5, n(\%)$ & $4(3)$ & $9(4)$ & $45(4)$ & 0.659 \\
\hline Overweight or obesity, $n(\%)$ & $132(85)$ & $175(85)$ & 961 (79) & 0.075 \\
\hline hs-CRP $\geq 2$ ng/L, $n(\%)$ & $30(19)$ & $21(10)$ & $87(7)$ & $<0.001$ \\
\hline Sinus rhythm, $n(\%)$ & $73(47)$ & $152(73)$ & $971(80)$ & $<0.001$ \\
\hline
\end{tabular}

The numbers in parentheses mean percentages (for categorical variables) or interquartile intervals (for continuous variables). $C A D$, coronary artery disease; $C K D$, chronic kidney disease; $h s-C R P$, high-sensitivity C-reactive protein; $L V E F$, left ventricular ejection fraction; $M I$, myocardial infarction; $P C I$, percutaneous coronary intervention

Patients underwent percutaneous coronary angioplasty and stenting or CABG surgery at the discretion of treating clinicians. Coronary procedure-related MI (type 4 and 5 MI) was defined according to current diagnostic criteria [19].

\section{Follow-up}

Patients were followed over time in a dedicated outpatient clinic and managed as clinically indicated. Follow-up data were retrieved in May 2020 from electronic health records and phone calls to patients or their relatives. For patients who died in a hospital or at home, the cause of death was elucidated from the medical records or the local physician who signed the death certificate. The attribution of cardiovascular death required documentation of significant arrhythmias or cardiac arrest, or death attributable to HF or MI in the absence of any other precipitating factor. The primary endpoint was all-cause death. The secondary endpoints were (1) a composite of cardiovascular death or nonfatal MI, and (2) cardiovascular death, non-fatal MI, or late revascularization (over 90 days from enrolment, excluding cases of MI) [20]. When multiple events occurred, patients were censored at the time of the first event. Follow-up events were adjudicated by an independent trained investigator, blinded to MPS data and coronary anatomy. No patient was lost to follow-up.

\section{Statistical analysis}

The statistical analysis was carried out using SPSS version 25.0 (IBM Corp., Armonk, NY) and R 3.6.2 (R Foundation for Statistical Computing, Vienna, Austria). Normal distribution was assessed by plotting a histogram and running the Kolmogorov-Smirnov test; continuous variables were expressed as mean and 95\% confidence interval, and non-normally distributed variables as median and interquartile interval. Mean differences among groups were evaluated through the unpaired Student $t$ test, the MannWhitney $U$ test, or the Kruskal-Wallis one-way analysis of variance, as appropriate. Categorical variables were compared by the chi-square test with Yates correction. The strength of correlations was evaluated through the Spearman rank correlation method. The best cut-off at receiver operating characteristics analysis was searched through the Youden method. Estimates of the cumulative event rate were calculated by the unadjusted Kaplan-Meier method and log-rank tests were used to test for differences between curves. Predictors of survival were searched through multivariable Cox regression analysis. The prognostic model included only 2 variables (the number of diseased vessels and early revascularization) to satisfy the one-in-ten event rule [21]. Two-tailed $p$ values $<0.05$ were considered significant.

\section{Results}

\section{Patient characteristics and MPS results}

The main characteristics of patients, stratified across categories of LVEF ( $\geq 50 \%, 36-49 \%, \leq 35 \%$ ), are reported in Table 1. Patients with severe systolic dysfunction (LVEF $\leq 35 \%$ ) were oldest, most often men, and with the highest likelihood of prior elective or urgent coronary 
Table 2 Findings from myocardial perfusion scintigraphy (MPS) and anatomical imaging

\begin{tabular}{|c|c|c|c|c|}
\hline & $\begin{array}{l}\mathrm{LVEF} \leq 35 \% \\
n=156(10 \%)\end{array}$ & $\begin{array}{l}\text { LVEF } 36-49 \% \\
n=207(13 \%)\end{array}$ & $\begin{array}{l}\mathrm{LVEF} \geq 50 \% \\
n=1213(77 \%)\end{array}$ & $p$ \\
\hline \multicolumn{5}{|l|}{ MPS } \\
\hline Exercise, dipyridamole, dobutamine, $n(\%)$ & $93,63,0(60,40,0)$ & $128,77,0(62,37,0)$ & $853,358,0(70,30,0)$ & 0.004 \\
\hline LVEF rest $(\%)$ & $30(26-34)$ & $43(40-46)$ & $63(58-69)$ & $<0.001$ \\
\hline LVEF stress (\%) & $30(26-35)$ & $41(37-45)$ & $62(56-69)$ & $<0.001$ \\
\hline LVEDVi rest $\left(\mathrm{mL} / \mathrm{m}^{2}\right)$ & $91(78-113)$ & $71(60-82)$ & $47(39-55)$ & $<0.001$ \\
\hline LVEDVi stress $\left(\mathrm{mL} / \mathrm{m}^{2}\right)$ & $91(79-115)$ & $71(60-86)$ & $46(39-55)$ & $<0.001$ \\
\hline LVESVi rest $\left(\mathrm{mL} / \mathrm{m}^{2}\right)$ & $63(53-79)$ & $40(34-48)$ & $17(13-23)$ & $<0.001$ \\
\hline LVESVi stress $\left(\mathrm{mL} / \mathrm{m}^{2}\right)$ & $64(53-84)$ & $42(34-52)$ & $18(12-23)$ & $<0.001$ \\
\hline $\operatorname{LVMi}\left(\mathrm{g} / \mathrm{m}^{2}\right)$ & $98(85-111)$ & $82(73-93)$ & $70(64-78)$ & $<0.001$ \\
\hline PFR rest $\left(\mathrm{s}^{-1}\right)$ & $1.40(1.06-2.03)$ & $2.11(1.56-2.66)$ & $2.43(2.08-2.83)$ & $<0.001$ \\
\hline PFR stress $\left(\mathrm{s}^{-1}\right)$ & $1.56(1.10-2.04)$ & $2.06(1.57-2.51)$ & $2.44(2.03-2.99)$ & $<0.001$ \\
\hline Rest RPP (b.p.m.*mmHg) & $9300(7800-11,250)$ & $9400(8200-11,050)$ & $9400(8100-11,200)$ & 0.974 \\
\hline Peak RPP (b.p.m.*mmHg) & $15,500(11700-20,800)$ & $15,800(11800-21,000)$ & $19,000(13725-24,300)$ & $<0.001$ \\
\hline Peak workload (W) & $100(75-125)$ & $100(75-125)$ & $100(100-125)$ & 0.218 \\
\hline Rest HR (b.p.m.) & $70(65-84)$ & $68(62-75)$ & $65(62-75)$ & 0.130 \\
\hline Peak HR (b.p.m.) & $78(62-88)$ & $81(70-88)$ & $80(71-88)$ & 0.883 \\
\hline$\%$ of predicted peak HR $(\%)$ & $78(61-81)$ & $79(70-85)$ & $80(73-85)$ & 0.024 \\
\hline Maximal stress test, $n(\%)$ & $15(10)$ & $62(30)$ & $485(40)$ & $<0.001$ \\
\hline Positive test, $n(\%)$ & $25(16)$ & $39(19)$ & $164(14)$ & 0.009 \\
\hline Test on beta-blockers, $n(\%)$ & $57(37)$ & $20(10)$ & $42(3)$ & $<0.001$ \\
\hline SRS & $11(5-18)$ & $6(3-10)$ & $1(0-3)$ & $<0.001$ \\
\hline SSS & $15(10-22)$ & $12(7-17)$ & $6(4-10)$ & $<0.001$ \\
\hline SDS & $4(1-7)$ & $6(3-8)$ & $4(3-7)$ & 0.002 \\
\hline $\mathrm{SDS}<4, n(\%)$ & $70(45)$ & $62(30)$ & $428(35)$ & 0.012 \\
\hline $\mathrm{SDS} \geq 7, n(\%)$ & $41(26)$ & $78(38)$ & $344(28)$ & 0.017 \\
\hline \multicolumn{5}{|l|}{ Invasive coronary angiography } \\
\hline Significant stenoses in LMC, $n(\%)$ & $3(2)$ & $4(2)$ & $15(1)$ & 0.380 \\
\hline Significant CAD (epicardial arteries/LMC), $n(\%)$ & $82(53)$ & $161(78)$ & $724(60)$ & $<0.001$ \\
\hline $1-, 2-, 3$-vessel disease, $n(\%)$ & $34,23,25(22,15,16)$ & $76,49,36(37,24,17)$ & $437,190,97(36,16,8)$ & $<0.001$ \\
\hline Multivessel CAD, $n(\%)$ & $48(31)$ & $85(41)$ & $287(24)$ & $<0.001$ \\
\hline Significant stenoses in LAD, $n(\%)$ & $113(72)$ & $128(62)$ & $478(39)$ & $<0.001$ \\
\hline Significant stenoses in LCx, $n(\%)$ & $88(56)$ & $92(44)$ & $411(34)$ & $<0.001$ \\
\hline Significant stenoses in RCA, $n(\%)$ & $56(36)$ & $97(47)$ & $362(30)$ & $<0.001$ \\
\hline
\end{tabular}

The numbers in parentheses mean percentages (for categorical variables) or interquartile intervals (for continuous variables). $C A D$, coronary artery disease; $H R$, heart rate; $L A D$, left anterior descending coronary artery; $L C x$, left circumflex coronary artery; $L V E D V i$, left ventricular end-diastolic volume index; $L V E F$, left ventricular ejection fraction; $L V E S V i$, left ventricular end-systolic volume index; $L V M i$, left ventricular mass index; $P F R$, peak filling rate; $R C A$, right coronary artery; $R P P$; rate-pressure product; $S D S$, summed difference score; $S R S$, summed rest score; SSS, summed stress score

revascularization. As expected, these patients had also the greatest impairment of LV geometry, systolic, and diastolic function (Table 2). Additionally, patients with LVEF $\leq 35 \%$ had the highest frequency of significant $\mathrm{CAD}$ and a greater number of diseased vessels (Table 2).

In the whole population, SRS and SSS displayed significant inverse correlations with LVEF and direct correlations with LV volumes, despite a great dispersion of data. Conversely, no correlation with SDS emerged
(Supplemental Fig. 1). Accordingly, median SSS values increased with decreasing LVEF, being were 6 in patients with LVEF $\geq 50 \%, 12$ in those with LVEF 36 to $49 \%$, and 15 in those with LVEF $\leq 35 \%$, and median SRS values were 1,6 , and 11 in the same groups (Table 2). Patients with LVEF $\leq 35 \%$ had no significant inducible ischemia (SDS <4) in $45 \%$ of cases and were those least likely to have extensive inducible ischemia (SDS $\geq 7$ ) (Table 2). 
Table 3 Revascularization and outcomes

\begin{tabular}{|c|c|c|c|c|}
\hline & $\begin{array}{l}\mathrm{LVEF} \leq 35 \% \\
n=156(10 \%)\end{array}$ & $\begin{array}{l}\text { LVEF } 36-49 \% \\
n=207(13 \%)\end{array}$ & $\begin{array}{l}\mathrm{LVEF} \geq 50 \% \\
n=1213(77 \%)\end{array}$ & $p$ \\
\hline \multicolumn{5}{|l|}{ Early revascularization } \\
\hline Revascularization, $n(\%)$ & $38(24)$ & $107(69)$ & $541(45)$ & $<0.001$ \\
\hline PCI, $n(\%)$ & $26(17)$ & $92(59)$ & $495(41)$ & $<0.001$ \\
\hline $\mathrm{CABG}, n(\%)$ & $12(8)$ & $15(10)$ & $47(4)$ & 0.019 \\
\hline Revascularization of LAD, $n(\%)$ & $29(19)$ & $78(38)$ & $304(25)$ & 0.001 \\
\hline Revascularization of LCx, $n(\%)$ & $8(5)$ & $34(16)$ & $184(15)$ & 0.054 \\
\hline Revascularization of RCA, $n(\%)$ & $10(6)$ & $35(17)$ & $177(15)$ & 0.149 \\
\hline \multicolumn{5}{|l|}{ Therapy at discharge } \\
\hline Aspirin, $n(\%)$ & $140(90)$ & $176(85)$ & $802(66)$ & $<0.001$ \\
\hline Statin, $n(\%)$ & $94(60)$ & $107(52)$ & $552(46)$ & 0.004 \\
\hline $\mathrm{ACEi} / \mathrm{ARB}, n(\%)$ & $113(72)$ & $130(63)$ & $748(62)$ & 0.106 \\
\hline Beta-blocker, $n(\%)$ & $150(96)$ & $140(68)$ & $569(47)$ & $<0.001$ \\
\hline $\mathrm{CCB}, n(\%)$ & $7(4)$ & $38(18)$ & $220(18)$ & $<0.001$ \\
\hline Nitrates, $n(\%)$ & $26(17)$ & $23(11)$ & $94(8)$ & 0.001 \\
\hline Diuretic, $n(\%)$ & $153(98)$ & $106(51)$ & $399(33)$ & $<0.001$ \\
\hline \multicolumn{5}{|l|}{ Outcomes } \\
\hline CV death or non-fatal MI, $n(\%)$ & $32(21)$ & $26(13)$ & $73(6)$ & $<0.001$ \\
\hline FU to CV death or non-fatal MI (years) & $2.5(1.3-5.3)$ & $4.9(2.7-7.0)$ & $4.6(2.8-6.0)$ & $<0.001$ \\
\hline Time to $C V$ death or non-fatal MI (years) & $1.8(1.5-3.2)$ & $1.7(0.7-2.9)$ & $1.8(0.7-3.3)$ & $<0.001$ \\
\hline Time to end FU (years) & $3.5(1.2-5.9)$ & $5.7(3.2-7.3)$ & $4.9(3.1-6.3)$ & $<0.001$ \\
\hline All-cause death, $n(\%)$ & $35(22)$ & $10(5)$ & $26(2)$ & $<0.001$ \\
\hline FU to all-cause death (years) & $2.5(1.3-5.4)$ & $5.2(3.0-7.2)$ & $4.6(2.8-6.1)$ & $<0.001$ \\
\hline Time to all-cause death (years) & $1.9(1.4-3.2)$ & $2.4(1.9-4.1)$ & $2.9(1.0-5.3)$ & $<0.001$ \\
\hline Time to end FU (years) & $2.7(1.2-5.9)$ & $4.9(2.7-7.0)$ & $4.7(2.9-6.1)$ & $<0.001$ \\
\hline $\mathrm{CV}$ death, non-fatal MI, late revascularization, $n(\%)$ & $37(24)$ & $50(24)$ & $218(18)$ & 0.001 \\
\hline FU to CV death, non-fatal MI, late revascularization (years) & $2.2(1.2-5.0)$ & $4.4(2.3-4.7)$ & $4.2(2.4-5.8)$ & $<0.001$ \\
\hline Time to CV death, non-fatal MI, late revascularization (years) & $1.8(1.1-3.2)$ & $1.7(0.6-3.2)$ & $1.7(0.5-3.2)$ & $<0.001$ \\
\hline Time to end FU (years) & $2.7(1.2-5.9)$ & $5.7(3.2-7.2)$ & $4.8(3.0-6.3)$ & $<0.001$ \\
\hline
\end{tabular}

The numbers in parentheses mean percentages (for categorical variables) or interquartile intervals (for continuous variables). Early revascularization was performed at the end of the diagnostic workup and within 3 months from myocardial perfusion scintigraphy. Late revascularization was defined as a revascularization event occurring after $>3$ months from the end of the baseline diagnostic workup. $A C E i / A R B$, angiotensin-converting enzyme inhibitor/ angiotensin receptor blocker; $C A B G$, coronary artery bypass grafting; $C C B$, calcium channel blocker; $C V$, cardiovascular; $F U$, follow-up; $L A D$, left anterior descending coronary artery; $L C x$, left circumflex coronary artery; $L V E F$, left ventricular ejection fraction; $M I$, myocardial infarction; $P C I$, percutaneous coronary intervention; $R C A$, right coronary artery

Table 4 Best cut-offs of summed rest score (SRS), summed stress score (SSS), and summed difference score (SDS) in patients with left ventricular ejection fraction $\leq 35 \%$ : Youden index analysis

\begin{tabular}{|c|c|c|c|c|c|c|c|c|c|}
\hline & \multicolumn{3}{|c|}{ CV death, non-fatal MI } & \multicolumn{3}{|c|}{ All-cause death } & \multicolumn{3}{|c|}{ CV death, non-fatal MI, late revascularization } \\
\hline & Best cut-off & Sensitivity & Specificity & Best cut-off & Sensitivity & Specificity & Best cut-off & Sensitivity & Specificity \\
\hline SRS & 23 & 22 & 93 & 23 & 20 & 90 & 23 & 19 & 93 \\
\hline SSS & 25 & 31 & 88 & 28 & 23 & 89 & 28 & 22 & 93 \\
\hline SDS & 7 & 34 & 75 & 7 & 37 & 77 & 7 & 41 & 77 \\
\hline
\end{tabular}

$C I$, confidence interval; $C V$, cardiovascular; $H R$, hazard ratio; $M I$, myocardial infarction 
Table 5 Independent prognostic value of summed rest score (SRS), summed stress score (SSS), summed difference score (SDS), and their best cut-offs in patients with left ventricular ejection fraction $\leq 35 \%$ : multivariable Cox regression analysis

Model: early revascularization, number of diseased vessels

\begin{tabular}{|c|c|c|c|c|c|c|c|c|c|}
\hline & \multicolumn{3}{|c|}{ CV death, non-fatal MI } & \multicolumn{3}{|c|}{ All-cause death } & \multicolumn{3}{|c|}{$\mathrm{CV}$ death, non-fatal MI, late revascularization } \\
\hline & HR & $95 \% \mathrm{CI}$ & $p$ & HR & $95 \% \mathrm{CI}$ & $p$ & HR & $95 \% \mathrm{CI}$ & $p$ \\
\hline SRS & - & - & 0.052 & - & - & 0.052 & - & - & 0.051 \\
\hline $\mathrm{SRS} \geq 23$ & 3.65 & $1.49-8.90$ & 0.004 & 2.93 & $1.22-7.05$ & 0.017 & 3.54 & $1.46-8.58$ & 0.005 \\
\hline SSS & 1.05 & $1.01-1.09$ & 0.017 & 1.05 & $1.01-1.09$ & 0.023 & 1.05 & $1.01-1.09$ & 0.011 \\
\hline $\mathrm{SSS} \geq 25$ & 4.48 & $1.92-10.41$ & 0.001 & & & & & & \\
\hline $\mathrm{SSS} \geq 28$ & & & & 3.35 & $1.44-7.80$ & 0.005 & 4.05 & $1.72-9.53$ & 0.001 \\
\hline SDS & - & - & 0.307 & - & - & 0.430 & - & - & 0.168 \\
\hline $\operatorname{SDS} \geq 7$ & - & - & 0.205 & - & - & 0.129 & - & - & 0.108 \\
\hline
\end{tabular}

The best cut-offs are reported in Table 4. Absolute summed rest score (SRS), summed stress score (SSS), and summed difference score (SDS) or their best cut-offs are evaluated in prognostic models including the occurrence of early revascularization and the number of vessels with significant stenoses. $C I$, confidence interval; $C V$, cardiovascular; $H R$, hazard ratio; $M I$, myocardial infarction

\section{Short-term follow-up: revascularization and medical treatment}

Twenty-four percent of patients with LVEF $\leq 35 \%$ underwent early revascularization, as compared to $52 \%$ of those with LVEF $36-49 \%$, and $45 \%$ of those with LVEF $\geq 50 \%$. CABG surgery was more commonly performed than in the other 2 LVEF categories, although less frequently than percutaneous coronary angiography (Table 3 ). Two patients had a peri-procedural MI, both of them belonging to the LVEF $\geq 50 \%$ group. The vast majority of patients with LVEF $\leq 35 \%$ were discharged on aspirin, beta-blocker, and diuretic therapy, while a lower number of patients were on statins. All these therapies were less often prescribed in patients with preserved LV systolic function (Table 3).

\section{Myocardial perfusion scintigraphy for risk prediction}

The number of events in the 3 LVEF categories is reported in Table 3. In the subset with LVEF $\leq 35 \%$, 32 cardiovascular death or non-fatal MI were recorded (23\%), 35 all-cause deaths (22\%), and 37 cardiovascular deaths, non-fatal MI, or late revascularizations $(27 \%)$. The survival free from all these endpoints was shorter than in the other 2 LVEF categories (Table 3 and Supplemental Fig. 2).

Among patients with LVEF $\leq 35 \%$, SRS, SSS, and SDS had very low AUC values for the prediction of the 3 endpoints (Fig. 1), and the best cut-offs were very high (Table 4). The number of patients above the cut-offs, with or without events, is reported in Supplemental Table 1. SRS and SSS and the corresponding cut-offs predicted a worse outcome in Cox regression models including the number of diseased vessels and early revascularization (Table 5, Fig. 2).

\section{Discussion}

In a large cohort of patients referred to MPS, those with LVEF $\leq 35 \%$ had a much greater extent of non-viable myocardium compared to those with better preserved systolic function, while the difference in severity of inducible ischemia was less prominent. Ischemia assessment has a lower prognostic significance in patients with severely reduced LVEF when compared to patients with normal or moderately reduced LVEF, as demonstrated by the lower AUC values. However, SRS and SSS cut-offs, albeit very high, predicted a worse outcome regardless of the number of diseased vessels and early revascularization (Central Illustration) (Fig. 3).

In the absence of other evidence, our results must be compared with findings from a post hoc analysis of the STICH trial, which had many limitations [22]. Notable differences from STICH were as follows: (1) the assessment of patients with LVEF $\leq 35 \%$ regardless of whether coronary anatomy was amenable to surgical revascularization, (2) the systematic use of SPECT imaging instead of a radionuclide stress test or dobutamine stress echocardiogram, (3) the assessment of the whole range of SDS values instead of the categorization according to the SDS $=4$ cut-off or the amount of ischemic myocardium, and also (4) the use of a new technology, such as a CZT camera, allowing better image quality. Furthermore, we assessed consecutive patients referred to MPS over a 9year time-span and considered also other endpoints. Despite these differences, our results regarding the prognostic impact of inducible ischemia are basically in agreement with those from STICH, as they confirm the modest prognostic value of inducible ischemia, in patients with $\mathrm{HF}$ and reduced EF. Indeed, SDS values had very low AUC values for outcome prediction ( 0.504 for the primary endpoint), and both SDS and 

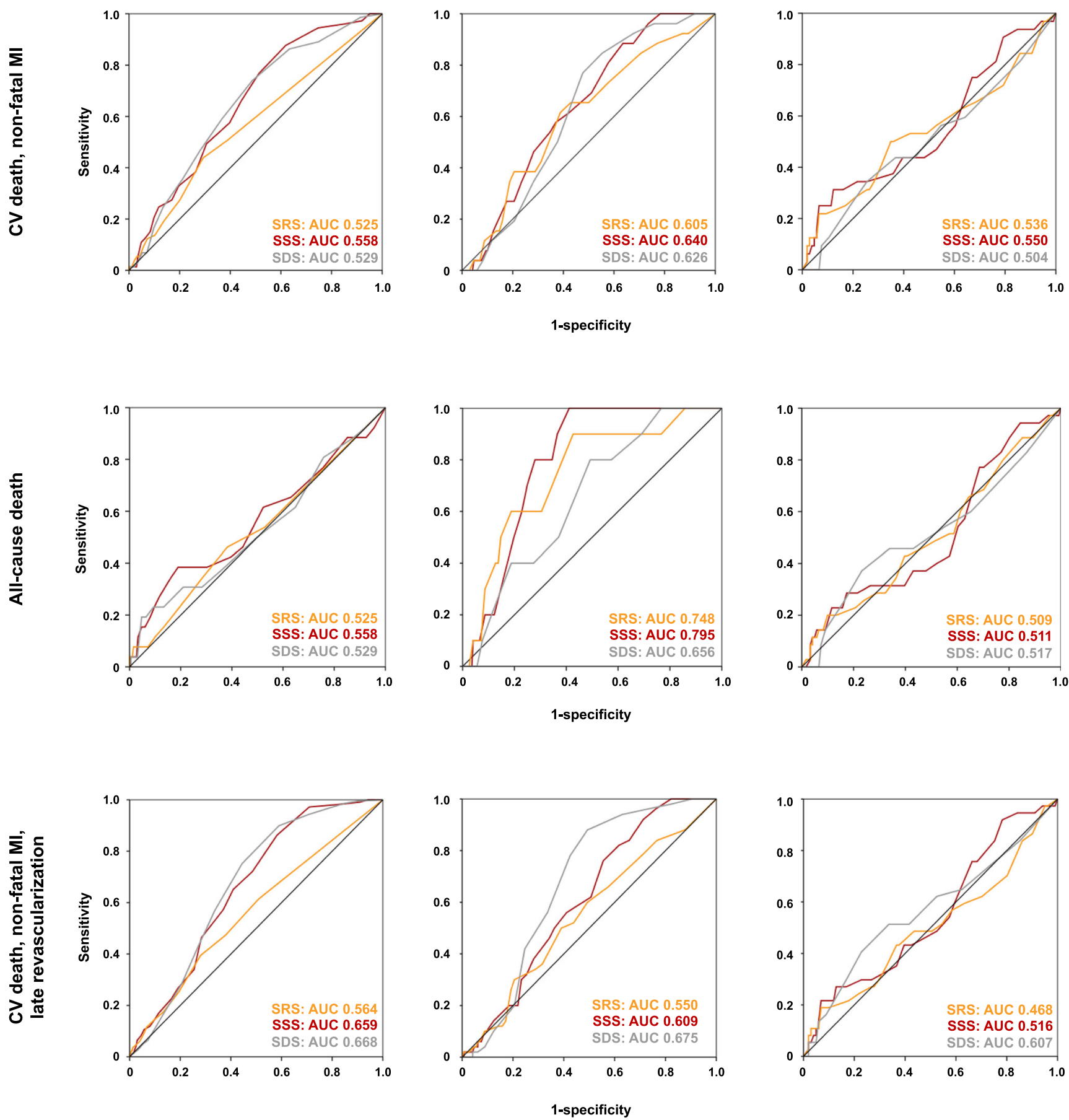

Fig. 1 Myocardial perfusion scintigraphy for outcome prediction. AUC, area under the curve; CV, cardiovascular; MI, myocardial infarction; SDS, summed difference score; SRS, summed rest score; SSS, summed stress score

the best cut-off ( $\mathrm{SDS}=7$ ) did not predict $\mathrm{CV}$ death or MI or $\mathrm{CV}$ death alone beyond the number of diseased vessels or the occurrence of revascularization, but only a composite outcome including late revascularization.

Patient prognosis worsened significantly only in patients with very large perfusion deficits at baseline, corresponding to large area of non-viable myocardium (SRS $\geq 23$ for $\mathrm{CV}$ death or MI, corresponding to $34 \%$ of LV mass). Similarly, the extent of perfusion deficits at peak stress (quantified by SSS values) was poorly predictive of outcome, except for very high SSS cut-offs (25 or 28 , corresponding to $37 \%$ or $41 \%$ of LV mass), above 
Fig. 2 Patient survival across left ventricular ejection fraction (LVEF) categories in patients undergoing or not undergoing early revascularization. $\mathrm{CV}$, cardiovascular

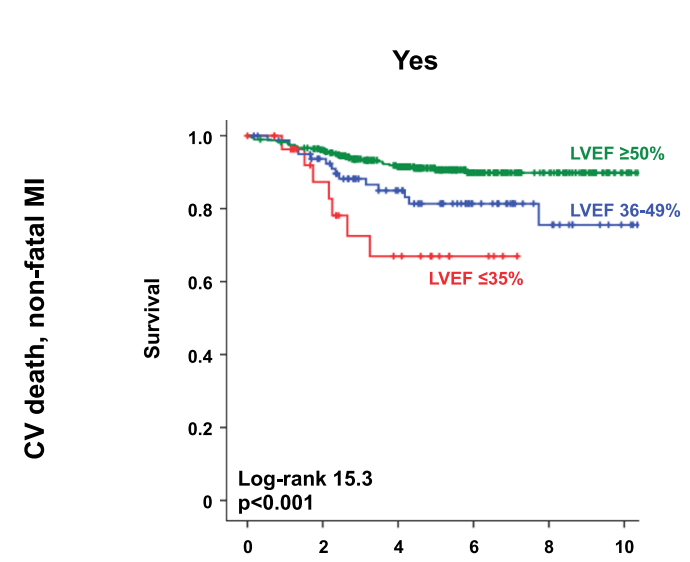

Revascularization

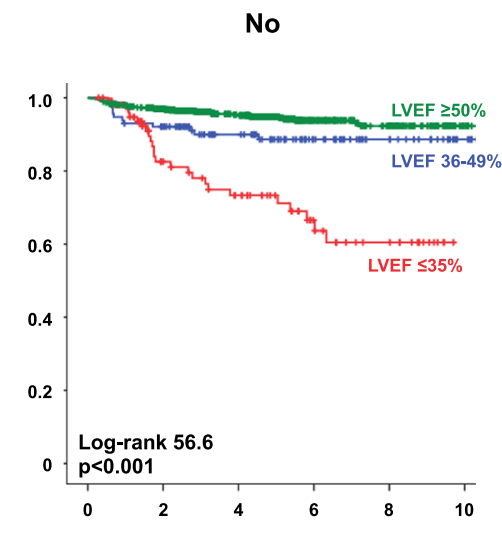

Years
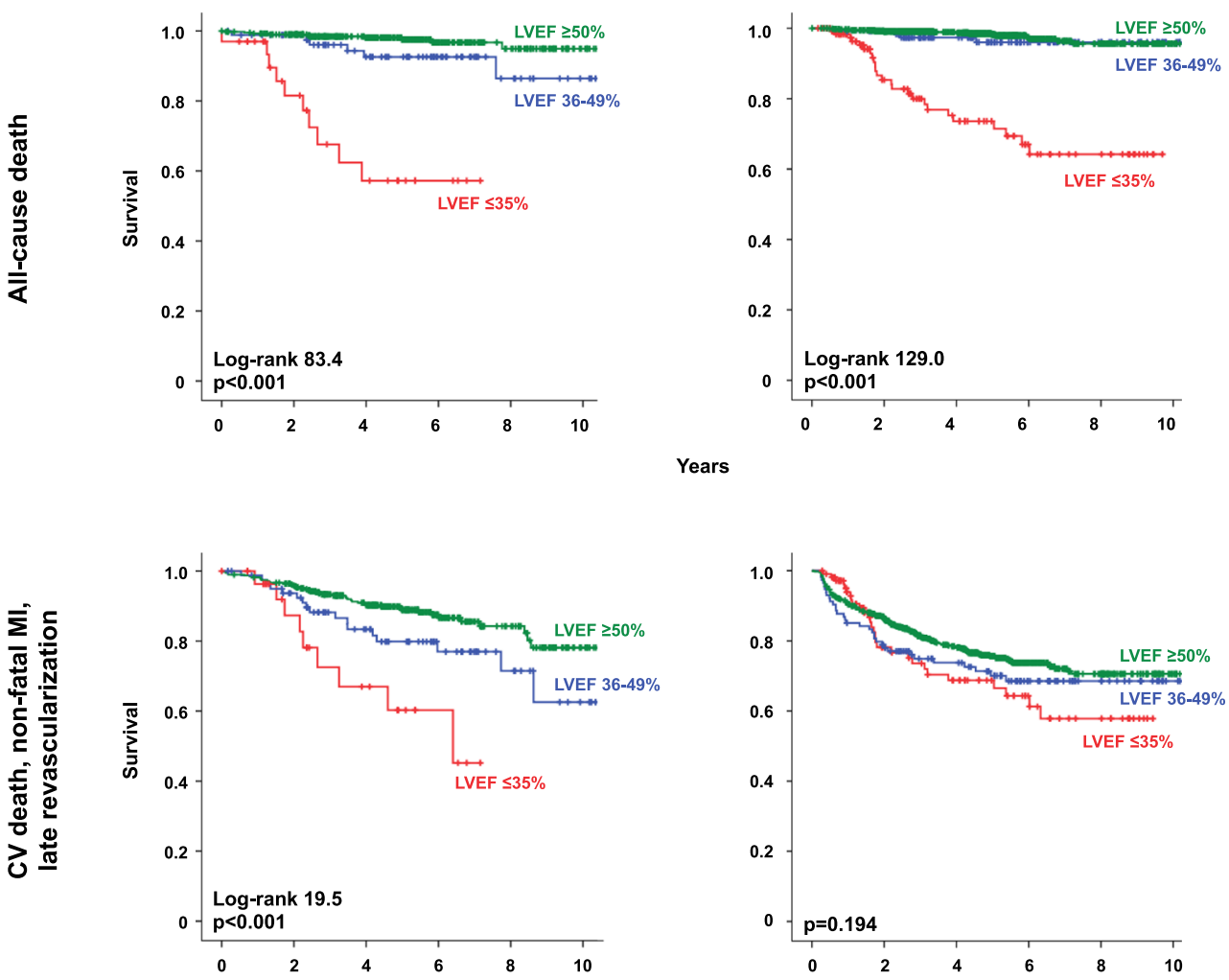

which patient outcome was apparently unaffected by the extent of CAD and even revascularization.

These results demonstrate that the search for ischemia testing is less effective for risk stratification in patients with $\mathrm{LVEF} \leq 35 \%$ than in those with better preserved LV function. A possible explanation could be related to the peculiar pathophysiology of HF with reduced EF, which includes several different main variables such as the activation of the renin-angiotensin-aldosterone axis and the sympathetic nervous system, with an increased risk of life-threatening ventricular arrhythmias [1]. According to this background, even the extent of ischemia and of viability could lose some of their prognostic meaning, although very large necrotic area still portends a worse outcome.

However, this study was not designed to elucidate the reasons of the lower prognostic yield of stress MPS in patients with $\mathrm{HF}$ and reduced EF, nor to shed further light on the controversial topic of the role of ischemia testing as a guide to revascularization. This last point could be best 

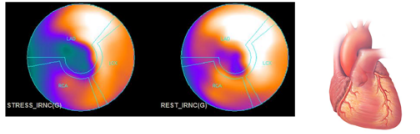

LVEF $\geq 50 \%$
Stress-rest SPECT for outcome prediction

in heart failure

LVEF $36-49 \%$

LVEF $\leq 35 \%$

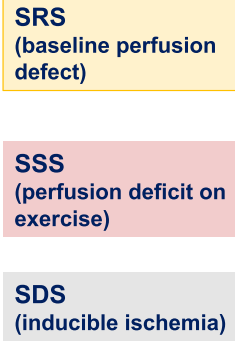

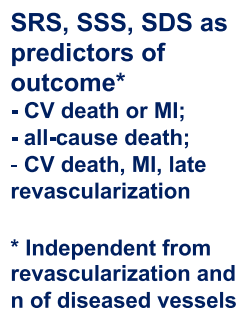

Fig. 3 Central Illustration. Findings from stress-rest single photon emission computed tomography (SPECT) across categories of left ventricular ejection fraction (LVEF). For details, see text. CAD, coronary artery

verified in the setting of a dedicated randomized controlled trial, which might consider global measures such as SDS, but should probably select higher cut-offs for revascularization. Indeed, we may consider that OMT confers a significant prognostic benefit in patients with $\mathrm{HF}$ and reduced ejection fraction, including those with ischemic etiology [23-29]; it is then reasonable to expect that revascularization might improve patient outcome over OMT only in patients with very large areas of viable but hypoperfused myocardium. However, the expected small additive benefit from revascularization warrants large, adequately powered study populations.

\section{Limitations}

Several study limitations must be acknowledged. The study protocol did not completely conform to the diagnostic flowchart of CCS [4]. Most notably, the systematic referral of patients to CCTA or ICA after MPS, and the use of the SDS $\geq 4$ cut-off to choose between CCTA and ICA represent deviations from the guideline-recommended protocol. Even the use of different SDS cut-offs, such as the ones previous proposed for risk stratification in a 20-segment model [30], might have led to different results. Nonetheless, our protocol allowed to search for ischemia (which "may be considered" in patients with LVEF $\leq 35 \%$ ) disease; CV, cardiovascular; MI, myocardial infarction; SDS, summed difference score; SRS, summed rest score; SSS, summed stress score

and to explore coronary anatomy to assess the feasibility of coronary revascularization (again in agreement with guidelines) [4]. Furthermore, there were no pre-established decisional criteria for revascularization of coronary artery stenoses $>70 \%$, and the need for revascularization of single lesions was established by the interventional cardiologist taking into account results from the assessment of myocardial viability and ischemia, according to common practice.

\section{Conclusions}

In patients with $\mathrm{LVEF} \leq 35 \%$, SRS and SSS are less predictive of outcome than in patients with better preserved systolic dysfunction, but very high cut-offs still retain independent prognostic significance from the number of vessels with significant stenoses and from early revascularization. The role of myocardial revascularization in this population remains uncertain.

Supplementary Information The online version contains supplementary material available at https://doi.org/10.1007/s00259-021-05312-4.

Author contribution AG, AA, and LS contributed to the study conception and design. Material preparation and data collection were performed by EMP, MAC, AC, and ME; analysis was performed by AA. The first draft of the manuscript was written by AG and all authors commented on previous versions of the manuscript. All authors read and approved the final manuscript. 


\section{Declarations}

Ethics approval The study conformed to the declaration of Helsinki and was approved by the Institution's human research committee.

Consent to participate All participants gave written informed consent.

Conflict of interest The authors declare no competing interests.

\section{References}

1. Metra M, Teerlink JR. Heart failure. Lancet. 2017;390:1981-95.

2. Gheorghiade M, Sopko G, De LL, et al. Navigating the crossroads of coronary artery disease and heart failure. Circulation. 2006;114: 1202-13.

3. Panza JA, Holly TA, Asch FM, et al. Inducible myocardial ischemia and outcomes in patients with coronary artery disease and left ventricular dysfunction. J Am Coll Cardiol. 2013;61:1860-70.

4. Knuuti J, Wijns W, Saraste A, et al. 2019 ESC guidelines for the diagnosis and management of chronic coronary syndromes. Eur Heart J. 2020;41:407-77.

5. Gimelli A, Pugliese NR, Buechel RR, et al. Changes in left ventricle myocardial volume during stress test using cadmium-zinc-telluride cardiac imaging: implications in coronary artery disease [published online ahead of print, 2019 Oct 24]. J Nucl Cardiol. 2019. https:// doi.org/10.1007/s12350-019-01930-6.

6. Gimelli A, Bottai M, Genovesi D, et al. High diagnostic accuracy of low-dose gated-SPECT with solid-state ultrafast detectors: preliminary clinical results. Eur J Nucl Med Mol Imaging. 2012;39:83-90.

7. Petretta M, Acampa W, Daniele S, et al. Long-term survival benefit of coronary revascularization in patients undergoing stress myocardial perfusion imaging. Circ J. 2016;80(2):485-93.

8. Gimelli A, Pugliese NR, Kusch A, et al. Accuracy of cadmiumzinc-telluride imaging in detecting single and multivessel coronary artery disease: is there any gender difference? Int $\mathrm{J}$ Cardiol. 2019;274:388-93.

9. Ponikowski P, Voors AA, Anker SD, et al. 2016 ESC guidelines for the diagnosis and treatment of acute and chronic heart failure: the task force for the diagnosis and treatment of acute and chronic heart failure of the European Society of Cardiology (ESC) developed with the special contribution of the Heart Failure Association (HFA) of the ESC. Eur Heart J. 2016;37:2129-200.

10. Yancy CW, Jessup M, Bozkurt B, et al. 2013 ACCF/AHA guideline for the management of heart failure: executive summary: a report of the American College of Cardiology Foundation/ American Heart Association Task Force on practice guidelines. Circulation. 2013;128:1810-52.

11. Velazquez EJ, Lee KL, Deja MA, et al. Coronary-artery bypass surgery in patients with left ventricular dysfunction. N Engl J Med. 2011;364:1607-16.

12. Sciagrà R, Bisi G, Santoro GM, Rossi V, Fazzini PF. Nitrate versus rest myocardial scintigraphy with technetium $99 \mathrm{~m}$-sestamibi: relationship of tracer uptake to regional left ventricular function and its significance in the detection of viable hibernating myocardium. Am J Card Imaging. 1995;9:157-66.

13. Giorgetti A, Marzullo P, Sambuceti G, et al. Baseline/post-nitrate Tc-99m tetrofosmin mismatch for the assessment of myocardial viability in patients with severe left ventricular dysfunction: comparison with baseline Tc-99m tetrofosmin scintigraphy/FDG PET imaging. J Nucl Cardiol. 2004;11:142-51.

14. Cerqueira MD, Weissman NJ, Dilsizian V, et al. Standardized myocardial segmentation and nomenclature for tomographic imaging of the heart. A statement for healthcare professionals from the Cardiac Imaging Committee of the Council on Clinical Cardiology of the American Heart Association. Circulation. 2002;105:539-42.

15. Ficaro EP, Lee BC, Kritzman JN, et al. Corridor4DM: the Michigan method for quantitative nuclear cardiology. J Nucl Cardiol. 2007;14:455-65.

16. Gimelli A, Rovai I, Liga R, et al. Appropriate use criteria in clinical routine practice: implications in a nuclear cardiology lab. Int J Card Imaging. 2016;32:1003-9.

17. Acampa W, Buechel RR, Gimelli A. Low dose in nuclear cardiology: state of the art in the era of new cadmium-zinc-telluride cameras. Eur Heart J Cardiovasc Imaging. 2016;17:591-5.

18. Gimelli A, Liga R, Duce V, et al. Accuracy of myocardial perfusion imaging in detecting multivessel coronary artery disease: a cardiac CZT study. J Nucl Cardiol. 2017;24:687-95.

19. Thygesen K, Alpert JS, Jaffe AS, et al. Fourth universal definition of myocardial infarction (2018). J Am Coll Cardiol. 2018;72:223164.

20. Neglia D, Liga R, Caselli C, et al. Anatomical and functional coronary imaging to predict long-term outcome in patients with suspected coronary artery disease: the EVINCI-outcome study. Eur Heart J Cardiovasc Imaging. 2019:jez248.

21. Vittinghoff E, McCulloch CE. Relaxing the rule of ten events per variable in logistic and Cox regression. Am J Epidemiol. 2007;165: 710-8.

22. Buckberg GD, Athanasuleas CL. The STICH trial: misguided conclusions. J Thorac Cardiovasc Surg. 2009;138:1060-1064.e2.

23. Garg R, Yusuf S. Overview of randomized trials of angiotensinconverting enzyme inhibitors on mortality and morbidity in patients with heart failure. JAMA. 1995;273:1450-6.

24. Packer M, Bristow MR, Cohn JN, et al. The effect of carvedilol on morbidity and mortality in patients with chronic heart failure. $\mathrm{N}$ Engl J Med. 1996;334:1349-55.

25. Effect of metoprolol CR/XL in chronic heart failure: Metoprolol $\mathrm{CR} / \mathrm{XL}$ Randomised Intervention Trial in Congestive Heart Failure (MERIT-HF). Lancet. 1999;353:2001-7.

26. CIBIS-II Investigators and Committees. The Cardiac Insufficiency Bisoprolol Study II (CIBIS-II): a randomised trial. Lancet. 1999;353:9-13.

27. Pitt B, Zannad F, Remme WJ, et al. The effect of spironolactone on morbidity and mortality in patients with severe heart failure. N Engl J Med. 1999;341:709-17.

28. McMurray JJ, Packer M, Desai AS, et al. Angiotensin-neprilysin inhibition versus enalapril in heart failure. N Engl J Med. 2014;371: 993-1004.

29. McMurray JJV, Solomon SD, Inzucchi SE, et al. Dapagliflozin in patients with heart failure and reduced ejection fraction. $\mathrm{N}$ Engl $\mathrm{J}$ Med. 2019;381:1995-2008.

30. Berman DS, Abidov A, Kang X, et al. Prognostic validation of a 17segment score derived from a 20-segment score for myocardial perfusion SPECT interpretation. J Nucl Cardiol. 2004;11:414-23.

Publisher's note Springer Nature remains neutral with regard to jurisdictional claims in published maps and institutional affiliations. 